\title{
Clinical presentation of anaplastic large-cell lymphoma in the central nervous system
}

\author{
MASASHI NOMURA $^{1}$, YOSHITAKA NARITA ${ }^{1}$, YASUJI MIYAKITA ${ }^{1}$, MAKOTO OHNO $^{1}$, \\ SHINTARO FUKUSHIMA ${ }^{2}$, TAKASHI MARUYAMA ${ }^{3}$, YOSHIHIRO MURAGAKI ${ }^{3}$ and SOICHIRO SHIBUI $^{1}$ \\ ${ }^{1}$ Department of Neurosurgery and Neuro-Oncology; ${ }^{2}$ Division of Pathology and Clinical Laboratories, National Cancer Center, \\ Tokyo 104-0045; ${ }^{3}$ Department of Neurosurgery, Tokyo Women's Medical University, Tokyo 116-8567, Japan
}

Received November 21, 2012; Accepted April 23, 2013

DOI: $10.3892 / \mathrm{mco} .2013 .110$

\begin{abstract}
The majority of primary central nervous system (CNS) lymphomas are diffuse large B-cell lymphomas (DLBCLs) and anaplastic large-cell lymphoma (ALCL) is a type of T-cell tumor that is rare in the CNS. The aim of this study was to elucidate the clinical presentation and standard therapy of ALCLs by investigating reported cases. Additionally, a case of anaplastic lymphoma kinase (ALK)-positive ALCL in a 20 -year-old man who exhibited no recurrence for $>5$ years following high-dose methotrexate (HD-MTX) treatment was described. Twenty-six immunocompetent patients with ALCL of the CNS that were previously reported and 1 case of ALCL of the CNS treated at our hospital were investigated. Overall survival (OS) was analyzed in relation to survival factors such as age, ALK status and the treatment regimen. The male:female ratio of the patients was 19:8. Of the 27 patients, 13 (48.1\%) were ALK-positive, 9 (33.3\%) were ALK-negative and the ALK status was not determined in the remaining 5 patients (18.5\%). ALK-positive ALCL occurred at a younger age (median age, 17 years) and exhibited a favorable course (5-year OS, 75.0\%), whereas ALK-negative ALCL presented
\end{abstract}

Correspondence to: Dr Yoshitaka Narita, Department of Neurosurgery and Neuro-Oncology, National Cancer Center, 5-1-1 Tsukiji, Chuo-ku, Tokyo 104-0045, Japan

E-mail: yonarita@ncc.go.jp

Abbreviations: CNS, central nervous system; PCNSL, primary central nervous system lymphoma; ALCL, anaplastic large-cell lymphoma; ALK, anaplastic lymphoma kinase; OS, overall survival; PS, performance status; LDH, lactate dehydrogenase; DLBCL, diffuse large B-cell lymphoma; HD-MTX, high-dose methotrexate; CHOP, cyclophosphamide + doxorubicin + vincristine + prednisolone; $\mathrm{NR}$, not reached; H\&E, hematoxylin and eosin; MRI, magnetic resonance imaging; FDG-PET, positron emission tomography with fluorodeoxyglucose

Key words: primary central nervous system lymphoma, anaplastic large-cell lymphoma, anaplastic lymphoma kinase, T-cell lymphoma at an older age (median age, 65 years) and resulted in fatal outcomes (5-year OS, <12.5\%). Similar to the findings for systemic ALCL, ALK positivity, age $<40$ years and chemotherapy are associated with long-term survival for ALCL of the CNS. Chemoradiotherapy including methotrexate is recommended for ALCL and the possibility of treatment with chemotherapy alone for ALK-positive ALCL is currently under consideration.

\section{Introduction}

Primary central nervous system (CNS) lymphoma (PCNSL) is the second most common malignant brain tumor, accounting for $2.9 \%$ of the primary brain tumors in Japan (1). Age, performance status (PS), lactate dehydrogenase (LDH) serum levels, cerebrospinal fluid protein concentrations and the involvement of deep structures of the brain are independent predictors of survival (2). The 2-year overall survival (OS) rate varies from 24 to $85 \%$ in proportion to the prognostic score based on the aforementioned risk factors (2). Although the majority of PCNSLs are diffuse large B-cell lymphomas (DLBCLs), primary T-cell lymphoma of the CNS accounts for only $2-8.5 \%$ of PCNSLs $(3,4)$. However, the survival of patients with primary T-cell lymphoma is similar to that of patients with B-cell PCNSL (5).

Anaplastic large-cell lymphoma (ALCL) is an uncommon type of T-cell lymphoma first reported by Stein et al (6). It is characterized by large pleomorphic CD30 (Ki-1)-expressing lymphoid blasts containing horseshoe-shaped nuclei (7). Currently, the fourth edition of the WHO classification of Tumors of Haematopoietic Lymphoid Tissues, published in 2008, divides systemic ALCLs into two entities: anaplastic lymphoma kinase (ALK)-positive and ALK-negative (8). In total, $60-85 \%$ of systemic ALCLs are ALK-positive lymphomas that exhibit the characteristic $\mathrm{t}(2 ; 5)(\mathrm{p} 23 ; \mathrm{q} 35)$ translocation that produces the ALK protein (7). This type is associated with a younger age of onset and a more favorable prognosis (9-11). Patients with ALK-positive ALCL are usually younger and have a better prognosis compared to those with ALK-negative ALCL. The 5-year OS rates are 80 and $40 \%$ in ALK-positive and -negative patients, respectively (7).

Although ALCL frequently involves lymph nodes and occasionally involves extranodal sites, such as the skin, soft 
tissues, bone, bone marrow, liver, lungs and gastrointestinal tract, it rarely occurs in the CNS $(9,12)$ and ALCL of the CNS is limited to case reports. A few case reviews suggested that although ALK positivity and younger age appear to be favorable prognostic factors, this disease is generally much more aggressive compared to systemic ALCL or PCNSL $(13,14)$. However, the precise prognosis and standard treatment have not yet been determined due to the small number of reported cases.

In this study, we reviewed previously published cases of ALCL and discussed the therapeutic management of ALCL of the CNS. We also report a case study of a patient with ALK-positive brain ALCL who underwent successful treatment with high-dose methotrexate (HD-MTX) alone and has not exhibited recurrence for $>5$ years.

\section{Materials and methods}

A search was conducted using PubMed for published studies in English on cases of immunocompetent patients with ALCL of the brain. The keywords used were 'anaplastic large-cell lymphoma', 'ALK' and 'primary central nervous system lymphoma'. Information regarding age, gender, location, ALK positivity, treatment and clinical course were collected from each study.

In addition to the 13 cases reviewed by George et al (13), an additional 13 reported cases (12-33) were selected and a total of 27 cases were summarized, including those of the present study (Table I). The survival time of 2 patients (cases 10 and 22) was not described, 13 patients had succumbed to the disease and 12 patients exhibited no evidence of disease at the time the studies were published.

The OS for 25 patients was calculated by the Kaplan-Meier survival method. Statistical analyses were performed using JMP software version 9 (SAS Institute Inc., Cary, NC, USA).

\section{Results}

Patient characteristics. Details of the 27 reported cases are provided in Table II. The male:female ratio was 19:8. The median age of the patients was 20 years (range, 4.0-82).

Of the 27 patients, 13 (48.1\%) were ALK-positive, $9(33.3 \%)$ were ALK-negative and the ALK status was not determined in the remaining 5 patients (18.5\%). Twenty-four patients expressed T-cell antigens and 3 patients had null-cell tumors (cases 1, 16 and 23). There was a difference in the age distribution between the ALK-positive and -negative groups. The ALK-positive tumors occurred in patients aged $<40$ years. Moreover, 10 out of the 13 ALK-positive patients (76.9\%) were aged $\leq 20$ years and the median age of these 13 patients was 17 years (range, 4.0-39 years). Conversely, ALK-negative tumors occurred in older patients. Out of the 9 ALK-negative patients, $8(88.9 \%)$ were $>40$ years of age and the median age of these 9 patients was 65 years (range, 22-82 years). No ALK-negative patient was $<20$ years of age.

A single lesion was observed in $13(48.1 \%)$ and multiple lesions in 12 patients $(44.4 \%)$, including 1 case of leptomeningeal metastasis (case 10).

The postoperative treatment was chemoradiotherapy for $14(51.9 \%)$, chemotherapy alone for $3(11.1 \%)$, radiotherapy alone for $6(22.2 \%)$ and supportive care alone for 4 patients
(14.8\%). In total, 17 patients $(63.0 \%)$ received some form of chemotherapy. The chemotherapy regimens of these 17 patients were as follows: MTX-based agents for 8 (47.1\%), cyclophosphamide + doxorubicin + vincristine + prednisolone (CHOP)-based agents for 4 (23.5\%), both MTX- and CHOP-based agents for $3(17.6 \%)$, other agents for $1(5.9 \%)$ and unknown for 1 patient $(5.9 \%)$.

Clinical courses and $O S$. The patients aged $<40$ years $(n=17)$ exhibited a longer OS compared to those aged $>40$ years $(n=8)$ and the median OS of the groups was not reached (NR) and 2.5 months, respectively $(\mathrm{P}<0.01, \log$-rank test; Fig. 1A), which demonstrated younger age as a good prognostic factor. The median OS of patients aged $\leq 20(n=13)$ and $>20(n=12)$ years was NR and 3.5 months, respectively $(\mathrm{P}=0.089)$.

In total, 10 out of the 13 ALK-positive patients (76.9\%) exhibited no evidence of disease at the time of case report publication. At least 4 out of the 13 ALK-positive patients (30.7\%), including our patient, exhibited no evidence of disease for $>5$ years after the initial diagnosis, whereas 6 out of the 9 ALK-negative patients $(66.7 \%)$ succumbed to the disease within 4 months after diagnosis. The median OS of each group was NR and 2 months, respectively ( $\mathrm{P}<0.01$; Fig. 1B). ALK was significantly correlated with the prognosis, with a 5 -year OS of 75.0 and $<12.5 \%$ in patients with ALK-positive $(n=12)$ and -negative ALCL $(n=8)$, respectively.

There was no difference in the OS patients with a single lesion $(n=12)$ and those with multiple lesions $(n=11 ; P=0.36$; Fig. 1C).

Chemotherapy improved patient survival, as the median OS with $(n=10)$ or without $(n=15)$ chemotherapy was NR and 2.5 months, respectively ( $\mathrm{P}=0.01$; Fig. 1D). Chemoradiotherapy tended to improve OS compared to radiotherapy alone $(\mathrm{P}=0.08)$; however, there was no difference in OS between patients who had received chemoradiotherapy and those who had received chemotherapy alone $(\mathrm{P}=0.73$, Fig. 1E). Three patients treated with chemotherapy alone had no evidence of disease until the time when their cases were reported and 2 patients them exhibited no recurrence for $>5$ years.

Case presentation. We have treated 90 cases of histologically proven PCNSL from 2000 to the present at our department, including 88 cases of DLBCL and 2 cases of T-cell lymphoma, including 1 ALCL. A 20-year-old immunocompetent man with no other significant medical history was hospitalized with generalized seizures. He did not have any neurological deficits and physical examination on admission revealed no abnormal findings. Complete blood counts and serum chemistries, including LDH and soluble interleukin-2 receptor levels, were normal. Magnetic resonance imaging (MRI) with gadolinium diethylenetriamine pentaacetic acid (Gd-DTPA) revealed a well-enhanced mass lesion in the left frontal lobe (Fig. 2A), with a surrounding high-intensity lesion on T2-weighted images, indicating significant edema. Whole-body CT scans with contrast medium revealed no abnormality. Based on the suspicion of high-grade glioma, awake brain surgery and gross total removal of the tumor were performed. No residual tumor was identified on postoperative MRI with Gd-DTPA. Pathological findings revealed the proliferation of large, atypical lymphocytes containing 


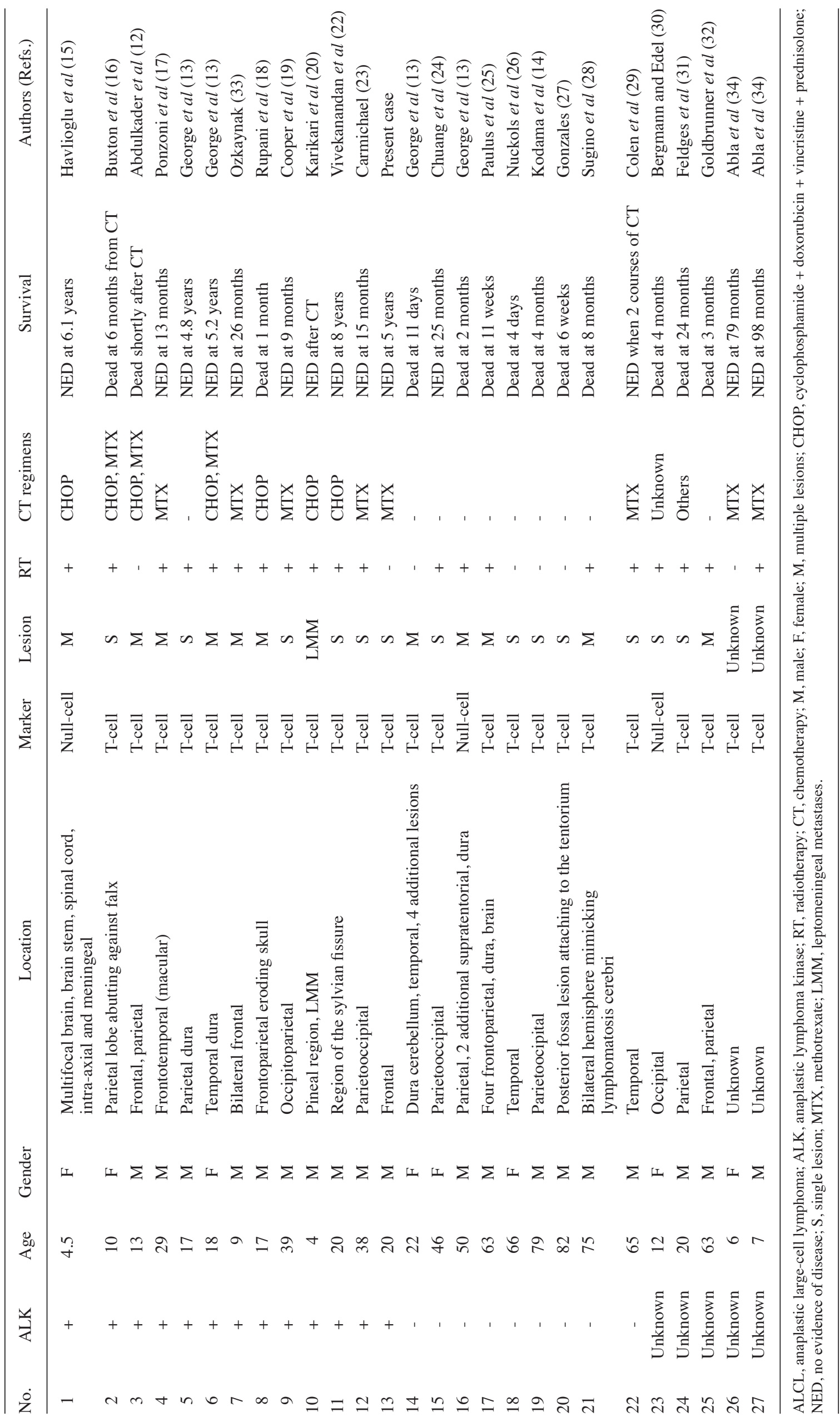


Table II. Summary of ALK lymphoma of reported cases.

\begin{tabular}{lccc}
\hline Variable & Total & ALK-positive & ALK-negative \\
\hline No. & 27 & 13 & 9 \\
Median age & 20.0 & 17.0 & 65.0 \\
(range) & $(4.0-82.0)$ & $(4.0-39.0)$ & $(22.0-82.0)$ \\
Gender (M:F) & $19: 8$ & $10: 3$ & $6: 3$ \\
Lesions & & & \\
Single & 13 & 6 & 5 \\
Multiple & 12 & 7 & 4 \\
Unknown & 2 & 0 & 0 \\
Initial therapy & & & \\
RT alone & 6 & 1 & 4 \\
RT+CT & 14 & 10 & 1 \\
CT alone & 3 & 2 & 0 \\
None & 4 & 0 & 4 \\
CT regimens & & & \\
MTX-based & 8 & 5 & 0 \\
CHOP-based & 4 & 4 & 0 \\
MTX+CHOP-based & 3 & 3 & 0 \\
Others & 1 & 0 & 0 \\
Unknown & 1 & 0 & \\
\hline ALK & & & \\
\hline
\end{tabular}

ALK, anaplastic lymphoma kinase; $\mathrm{M}$, male; F, female; RT, radiotherapy; $\mathrm{CT}$, chemotherapy; CHOP, cyclophosphamide + doxorubicin + vincristine + prednisolone; MTX, methotrexate.

scattered horseshoe-shaped nuclei on hematoxylin and eosin (H\&E)-stained slides (Fig. 2C). Immunohistochemistry revealed that the tumor cells were positive for CD3 and ALK-1 (Fig. 2D), but negative for CD20. This patient was finally diagnosed with ALK-1-positive ALCL. Bone marrow examination findings were normal.

Treatment was immediately initiated with intravenous HD-MTX $\left(3.5 \mathrm{~g} / \mathrm{m}^{2}\right)$. The patient completed 3 courses of HD-MTX, one every 2 weeks. Cerebral MRI and systemic positron emission tomography with fluorodeoxyglucose (FDG-PET) revealed no abnormal findings and radiotherapy was not performed. He had regular MRI examinations every 3 months until 3 years after the diagnosis and every 6 months thereafter. There has been no recurrence of the disease for 5 years (Fig. 2B) and the patient has exhibited no neurological deficits.

\section{Discussion}

We demonstrated that ALK-positive ALCL in the CNS presents at a younger age, has a good prognosis and is sensitive to chemoradiotherapy in the analysis of reported cases.

Shenkier et al (5) reported a retrospective analysis of patients with T-cell PCNSL diagnosed between 1983 and 2003 at 12 institutions in 7 countries. In that report, among 25 cases of T-cell PCNSL in which the pathology report was reviewed, 3 cases (12\%) exhibited the characteristics of ALCL. Thus, ALCL is a rare disease of the brain, for which the literature
Overall survival

A

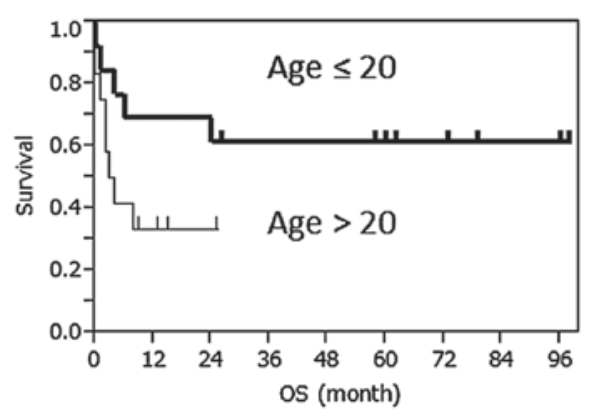

B

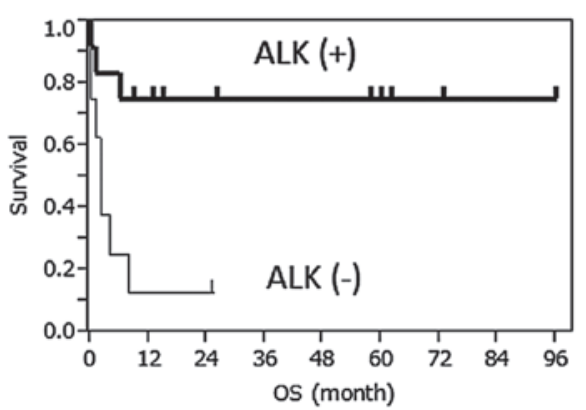

C

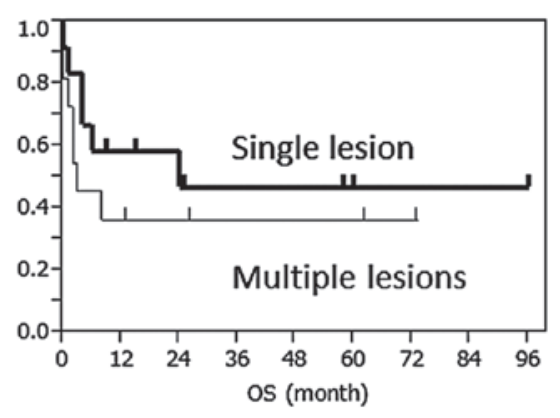

D

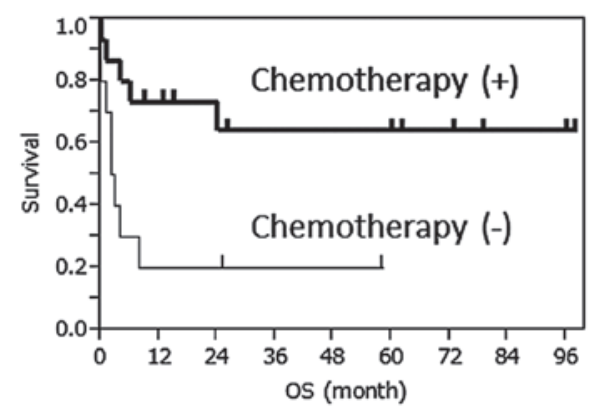

$\mathbf{E}$

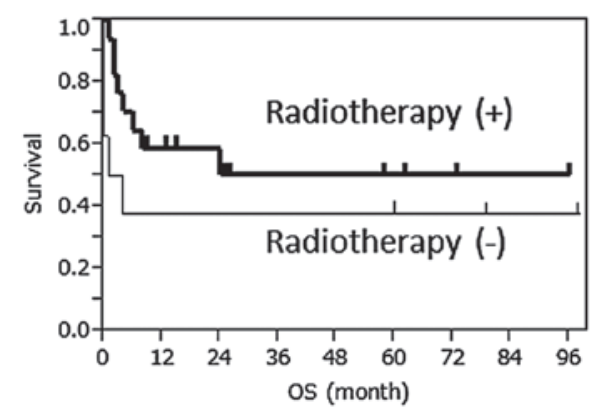

Figure 1. Kaplan-Meier survival curves for anaplastic large-cell lymphoma (ALCL) according to (A) age, (B) anaplastic lymphoma kinase (ALK) status, (C) number of lesions, (D) chemotherapy and (E) radiotherapy. 

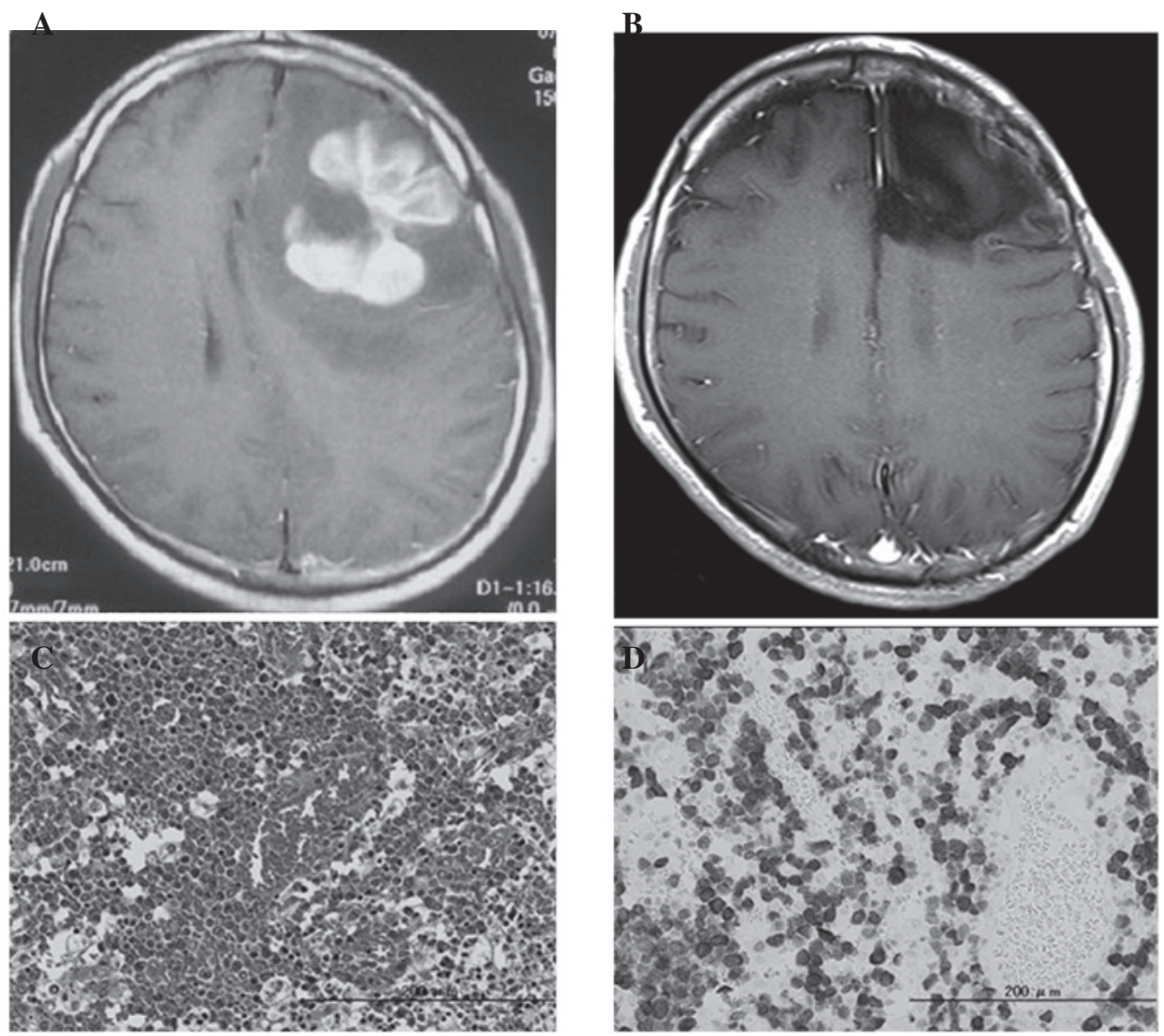

Figure 2. Preoperative magnetic resonance imaging (MRI) with (A) gadolinium diethylenetriamine pentaacetic acid (Gd-DTPA), (B) MRI 5 years after treatment, (C) hematoxylin and eosin (H\&E) staining and (D) immunohistochemistry for anaplastic lymphoma kinase 1 (ALK-1).

is limited to case reports. We collected twice as many cases as the largest reviews (13) and although our review was based on incomplete data from the literature, our findings may be of value in understanding this rare disease.

In general, ALCL of the CNS has been recognized to be significantly more aggressive compared to systemic ALCL or PCNSL $(13,14)$. However, our data demonstrate that ALK-positive ALCL has a good prognosis, similar to systemic ALCL $(10,11)$. Only 3 ALK-positive patients were reported to have succumbed to the disease. Tuberculosis or other infectious diseases of the CNS were suspected in these 3 patients (cases 2,3 and 8) at the initial presentation and they were treated with antituberculosis medications or antibiotics. Delayed diagnosis may result in a worse outcome compared to that observed in other ALK-positive patients. PCNSL is rare in adolescence (34) and the radiological findings of primary ALCL in the CNS often mimic those of infectious or immunological disease. However, an early diagnosis and the timely initiation of the appropriate treatment is critical.

There is no standard treatment for primary ALCL of the CNS. Our findings demonstrate the importance of chemoradiotherapy for ALCL. Of the 17 patients treated with chemotherapy, 11 received MTX-based agents. Moreover, 9 of these 11 patients exhibited no evidence of tumor at the time of case report publication, although 2 patients died shortly after treatment. As it is known that the standard chemotherapy regimen for PCNSL is MTX and that CHOP has not been proven to be effective against PCNSL $(35,36)$, MTX-based regimens are recommended for ALCL of the CNS. Our patient was treated with 3 courses of HD-MTX alone and he has exhibited no recurrence for $>5$ years. Abla et al (21) reported 10 pediatric cases of PCNSL, including 2 ALCLs treated with chemotherapy alone and the majority of the children achieved long-term remissions. Radiotherapy may decrease cognitive function and it is possible that ALK-positive patients, particularly younger patients, may need to start treatment with chemotherapy alone.

Our review also demonstrated that ALK-negative ALCL exhibits a poor prognosis and is very often fatal. The majority of ALK-negative patients were treated with radiotherapy or supportive care, due to their older age or poor PS. As ALK-negative ALCL tends to occur in older individuals, similar to PCNSL and DLBCL, chemoradiotherapy including HD-MTX should be initiated earlier.

In conclusion, our findings indicate that the prognosis of ALCL of the CNS is correlated with ALK positivity and patient age of $<40$ years. Chemoradiotherapy with MTX is recommended as the standard treatment for ALCL. However, additional multicenter studies including large numbers of cases are required.

\section{Acknowledgements}

This study was supported by National Cancer Center Research and Development Fund no. 55 


\section{References}

1. The committee of Brain Tumor Registry of Japan: Report of brain tumor registry of Japan (1984-2000) 12th edition. Neurol Med Chir (Tokyo) 49 Suppl: 1-101, 2009.

2. Ferreri AJ, Blay JY, Reni M, et al: Prognostic scoring system for primary CNS lymphomas: the International Extranodal Lymphoma Study Group experience. J Clin Oncol 21: 266-272, 2003.

3. Hayabuchi N, Shibamoto Y and Onizuka Y: Primary central nervous system lymphoma in Japan: a nationwide survey. Int J Radiat Oncol Biol Phys 44: 265-272, 1999.

4. Ferreri AJ, Reni M, Pasini F, et al: A multicenter study of treatment of primary CNS lymphoma. Neurology 58: 1513-1520, 2002.

5. Shenkier TN, Blay JY, O'Neill BP, et al: Primary CNS lymphoma of T-cell origin: a descriptive analysis from the internationa primary CNS lymphoma collaborative group. J Clin Oncol 23 2233-2239, 2005.

6. Stein H, Mason DY, Gerdes J, et al: The expression of the Hodgkin's disease associated antigen $\mathrm{Ki}-1$ in reactive and neoplastic lymphoid tissue: evidence that Reed-Sternberg cells and histiocytic malignancies are derived from activated lymphoid cells. Blood 66: 848-858, 1985

7. Jaffe ES, Harris NL, Stein H and Vardiman JW: Anaplastic large cell lymphoma. In: Pathology and Genetics: Tumors of Haematopoietic and Lymphoid Tissues. Jaffe ES, Harris NL, Stein H, Vardiman JW et al (eds). IARC Press, pp230-235, Lyon, 2001.

8. Swerdlow S, Campo E, Harris N, et al (eds): Anaplastic large cell lymphoma. WHO classification of Tumours of Haematopoietic and Lymphoid Tissues. 4th edition. IARC Press, pp312-319, Lyon, 2008.

9. Stein H, Foss HD, Durkop H, et al: CD30(+) anaplastic large cell lymphoma: a review of its histopathologic, genetic, and clinical features. Blood 96: 3681-3695, 2000.

10. Suzuki R, Kagami Y, Takeuchi K, et al: Prognostic significance of CD56 expression for ALK-positive and ALK-negative anaplastic large-cell lymphoma of T/null cell phenotype. Blood 96: 2993-3000, 2000.

11. Falini B, Pileri S, Zinzani PL, et al: ALK+ lymphoma: clinico-pathological findings and outcome. Blood 93: 2697-2706, 1999.

12. Abdulkader I, Cameselle-Teijeiro J,Fraga M, Rodriguez-Nunez A, Allut AG and Forteza J: Primary anaplastic large cell lymphoma of the central nervous system. Hum Pathol 30: 978-981, 1999.

13. George DH, Scheithauer BW, Aker FV, et al: Primary anaplastic large cell lymphoma of the central nervous system: prognostic effect of ALK-1 expression. Am J Surg Pathol 27: 487-493, 2003.

14. Kodama K, Hokama M, Kawaguchi K, Tanaka Y and Hongo K: Primary ALK-1-negative anaplastic large cell lymphoma of the brain: case report and review of the literature. Neuropathology 29: 166-171, 2009

15. Havlioglu N, Manepalli A, Galindo L, Sotelo-Avila C and Grosso L: Primary Ki-1 (anaplastic large cell) lymphoma of the brain and spinal cord. Am J Clin Pathol 103: 496-499, 1995.

16. Buxton N, Punt J and Hewitt M: Primary Ki-1-positive T-cell lymphoma of the brain in a child. Pediatr Neurosurg 29: 250-252, 1998.

17. Ponzoni M, Terreni MR, Ciceri F, et al: Primary brain CD30+ ALK1+ anaplastic large cell lymphoma ('ALKoma'): the first case with a combination of 'not common' variants. Ann Oncol 13: $1827-1832,2002$

18. Rupani A, Modi C, Desai S and Rege J: Primary anaplastic large cell lymphoma of central nervous system - a case report. J Postgrad Med 51: 326-327, 2005.
19. Cooper PB, Auerbach A, Aguilera NS, et al: Rare primary CNS anaplastic large cell lymphoma in an immunocompetent adult: a clinical-pathologic case report and review case of the literature. Clin Neuropathol 25: 232-236, 2006.

20. Karikari IO, Thomas KK, Lagoo A, Cummings TJ and George TM: Primary cerebral ALK-1-positive anaplastic large cell lymphoma in a child. Case report and literature review. Pediatr Neurosurg 43: 516-521, 2007.

21. Abla O, Sandlund JT, Sung L, et al: A case series of pediatric primary central nervous system lymphoma: favorable outcome without cranial irradiation. Pediatr Blood Cancer 47: 880-885, 2006.

22. Vivekanandan S, Dickinson P, Bessell E and O'Connor S: An unusual case of primary anaplastic large cell central nervous system lymphoma: an 8-year success story. BMJ Case Rep: Feb 24,2011 (Epub ahead of print). doi: 10.1136/bcr.11.2010.3550.

23. Carmichael MG: Central nervous system anaplastic large cell lymphoma in an adult: successful treatment with a combination of radiation and chemotherapy. Mil Med 172: 673-675, 2007.

24. Chuang SS, Huang W, Lin CN, et al: Primary cerebral anaplastic large cell lymphoma containing abundant reactive histiocytes and eosinophils. A case report and literature review. Pathol Res Pract 197: 647-652, 2001.

25. Paulus W, Ott MM, Strik H, Keil V and Muller-Hermelink HK: Large cell anaplastic (KI-1) brain lymphoma of T-cell genotype. Hum Pathol 25: 1253-1256, 1994.

26. Nuckols JD, Liu K, Burchette JL, McLendon RE and Traweek ST: Primary central nervous system lymphomas: a 30-year experience at a single institution. Mod Pathol 12: $1167-1173,1999$.

27. Gonzales M: Primary meningeal anaplastic large cell lymphoma. Pathology 35: 451-452, 2003.

28. Sugino T, Mikami T, Akiyama Y, Wanibuchi M, Hasegawa T and Mikuni N: Primary central nervous system anaplastic large-cell lymphoma mimicking lymphomatosis cerebri. Brain Tumor Pathol: Mar 18, 2012 (Epub ahead of print).

29. Colen CB, Rayes M, Kupsky WJ and Guthikonda M: Synchronous meningioma and anaplastic large cell lymphoma. Neuropathology 30: 260-266, 2010.

30. Bergmann $M$ and Edel G: Primary intracerebral non-Hodgkin's lymphoma. Pathologe 12: 246-253, 1991 (In German).

31. Feldges A, Gerhard L, Reinhardt V and Budach V: Primary cerebral anaplastic T-cell-lymphoma (type $\mathrm{Ki}-1$ ): review and case report. Clin Neuropathol 11: 55-59, 1992.

32. Goldbrunner R, Warmuth-Metz M, Tonn JC, Vince GH and Roosen K: Primary Ki-1-positive T-cell lymphoma of the brain - an aggressive subtype of lymphoma: case report and review of the literature. Surg Neurol 46: 37-41, 1996.

33. Ozkaynak MF: Favorable outcome of primary CNS anaplastic large cell lymphoma in an immunocompetent patient. J Pediatr Hematol Oncol 31: 128-130, 2009.

34. Abla $\mathrm{O}$ and Weitzman S: Primary central nervous system lymphoma in children. Neurosurg Focus 21: E8, 2006.

35. O'Neill BP, O'Fallon JR, Earle JD, Colgan JP, Brown LD and Krigel RL: Primary central nervous system non-Hodgkin's lymphoma: survival advantages with combined initial therapy? Int J Radiat Oncol Biol Phys 33: 663-673, 1995.

36. Schultz C, Scott C, Sherman W, et al: Preirradiation chemotherapy with cyclophosphamide, doxorubicin, vincristine, and dexamethasone for primary CNS lymphomas: initial report of radiation therapy oncology group protocol 88-06. J Clin Oncol 14: 556-564, 1996. 